\title{
ON SUPPORTLESS CONVEX SETS
}

\author{
J. M. BORWEIN ${ }^{1}$ AND D. W. TINGLEY
}

ABSTRACT. We give some general consiructions of supportless convex subsets of normed spaces and pose a number of open questions.

1. Introduction. In $1958 \mathrm{Klee}[5, \mathbf{1 5}, \mathbf{1 6}]$ gave an example of an incomplete linear subspace of $l_{2}(\mathbf{N})$ which contains a closed bounded (actually precompact) convex subset with no support points. He also asked whether such a subset could be found in a Banach space. Subsequently, Bishop and Phelps [1] showed that in a Banach space the support functionals of such a set were always norm dense in the dual space. This is the "dual" Bishop-Phelps theorem. In [2] they extended this to show that the support points were themselves norm dense in the boundary of the set - the Bishop-Phelps theorem. They also showed in [2] that the dual Bishop-Phelps theorem actually characterizes Banach spaces amongst normed spaces. In 1965 Bronsted and Rockafellar [6] published an elegant convex generalization (in terms of epsilon subgradients) of both Bishop-Phelps theorems simultaneously. In 1969 Peck [19], while studying more general support properties of convex subsets of topological vector spaces, showed that the Bishop-Phelps theorem failed in certain Fréchet spaces. In 1974, Ekeland [9] produced a far-ranging nonconvex extension of the Bishop-Phelps and Bronsted-Rockafellar results to metric spaces. This result is now called "Ekeland's variational principle".

Ekeland [9, 10], Sullivan [22], and Tuy [25] have surveyed the impressive variety of applications that the variational principle has already had. Sullivan [21] also observed that Ekeland's principle actually characterizes completeness of a metric space. When one specializes his construction to a normed setting, the function involved becomes continuous and convex and shows that the Bronsted-Rockafellar theorem (for continuous convex functions) characterizes Banach spaces.

The recent development of nonsmooth analysis [7] has substantially increased the interest in tangency properties of convex and nonconvex sets. Support properties play a critical role here. For example, if a normed space contains a supportless closed bounded convex set $C$, then two disjoint copies of $C$ produce an example of a closed bounded disconnected set $A$ whose tangent cone (in any of a number of definitions) at each point of $A$ is the whole space [3]. In such a setting much of the

Received by the editors February 5, 1984 and, in revised form, April 11, 1984. 1980 Mathematics Subject Classification. Primary 52A07: Secondary 46A55, 46B20. Key words and phrases. Support points, Bishop-Phelps theorem, convex sets.

${ }^{1}$ Research partially supported by a N.S.E.R.C. operating grant. 
more delicate theory of convex and nonsmooth analysis falls apart. For this and other reasons one wishes to know which normed spaces contain supportless sets. In the next two sections we sketch some positive results and indicate a series of open questions regarding the existence of supportless sets. Loosely speaking, we are searching for a converse to the Bishop-Phelps theorem.

2. Supportless convex sets. A subset $A$ of a topological vector space will be called supportless if no continuous linear functional achieves its bounds on $A$. Our main conjecture is that a normed space $X$ contains a closed bounded convex supportless set if and only if $X$ is incomplete. This might be compared with James' theorem [14], which shows that there is a continuous linear functional which fails to support the unit ball if and only if $X$ is nonreflexive. Our evidence for the conjecture is that we can construct supportless sets in a wide variety of types of incomplete normed spaces. Peck's technique in [19], which is to inductively apply James' theorem, seems, intrinsically, not to apply in a normed setting. The only remaining techniques seem to devolve to generalizations of Klee's original example [15]. An abstract form of this example is as follows.

THEOREM 1. Let $E$ be a Banach space and let $T: l_{2}(S) \rightarrow E$ be a bounded linear mapping such that $T$ is injective with proper norm-dense range. Suppose that $M \subset l_{2}(S)$ is a dense subspace with

$$
M \cap R\left(T^{*}\right)=0
$$

Then $C \subset E$, defined by

$$
C:=T(B), \quad B:=\left\{x \in l_{2}(S) \mid\|x\|_{2} \leqslant 1\right\},
$$

is a symmetric convex closed bounded subset of $E$ (which is precompact when $T$ is compact) such that (i) $C$ has no support points in $T(M)$, and (ii) $C \cap T(M)$ is supportless in $T(M)$.

Proof. Suppose $0 \neq \Phi \in(T(M))^{*}=E^{*}$, and suppose that $\Phi$ supports $C \cap$ $T(M)$ at $c:=T(b) \in T(M)$. Since $C \cap T(M)=T(B \cap M)$ and $T$ is injective, it follows that $b \in B$, and so

$$
\begin{aligned}
0 & =\sup \langle\Phi, C \cap T(M)-T(b)\rangle=\sup \left\langle T^{*}(\Phi), B \cap M-b\right\rangle \\
& =\sup \left\langle T^{*}(\Phi), B-b\right\rangle .
\end{aligned}
$$

Thus,

$$
\left\|T^{*}(\Phi)\right\| \leqslant\left\langle T^{*}(\Phi), b\right\rangle \leqslant\left\|T^{*}(\Phi)\right\|\|b\| \leqslant\left\|T^{*}(\Phi)\right\|,
$$

and we must have

$$
T^{*}(\Phi)=b \in M \cap R\left(T^{*}\right)=0 .
$$

Since $T^{*}$ is injective, this is impossible. This establishes both (i) and (ii).

We will say that a space is unsupportable if it contains a closed bounded convex supportless subset. Otherwise, we say the space is supportable. This leads to the following corollaries. 
COROLlaRY 1. Every separable Banach space E contains uncountably many unsupportable dense subspaces, including continuous operator ranges.

Proof. Let $K_{i}: l_{2}(\mathbf{N}) \rightarrow l_{2}(\mathbf{N})(i=1,2)$ be injective continuous linear operators with dense disjoint ranges: $R\left(K_{1}\right) \cap R\left(K_{2}\right)=0$ [12, p. 273]. Let $J: l_{2}(\mathbf{N}) \rightarrow E$ be injective, continuous, and linear with dense range [13]. Then we apply Theorem 1 with $T:=J K_{1}^{*}$ and $M:=K_{2}(N)$, where $N$ is any dense subspace of $l_{2}(\mathbf{N})$. Now $T$ is injective with dense range and

$$
R\left(T^{*}\right) \cap M \subseteq R\left(K_{1}\right) \cap R\left(K_{2}\right)=0,
$$

and so $T(M)$ is unsupportable. Since $T(M)=J K_{1}^{*} K_{2}(N)$ and there are uncountably many choices for $N$, there are uncountably many choices for $T(M)$. Letting $N:=l_{2}(\mathbf{N})$ produces an operator range.

Klee's original idea was essentially to use $K_{1}\left(x_{n}\right):=x_{n} / 2^{n}$ on $l_{2}(\mathbf{N})$.

COROLlaRY 2. Every countable dimension normed space contains a precompact closed convex supportless set and, therefore, is unsupportable.

Proof. Let $P$ be the space and let $E$ be its completion. Let $K: l_{2}(\mathbf{N}) \rightarrow l_{2}(\mathbf{N})$ be an injective compact linear mapping, and let $M$ be dense and countable dimension in $l_{2}(\mathbf{N})$ with $R\left(K^{*}\right) \cap M=0$. (Again this is possible by [12, p. 273].) A Markusevich basis argument [13] allows one to produce a compact injective linear mapping $J$ : $l_{2}(\mathbf{N}) \rightarrow E$ with $J(K(M))=P$, since both $P$ and $K(M)$ are dense and countable dimension. Since $T:=J K$ and $M$ satisfy the hypotheses of Theorem 1 , it follows that $P=T(M)$ is unsupportable as required.

EXAMPLE 1. Corollary 2 shows that many explicit spaces fail to be supportable. These include (i) the polynomials on a bounded interval in either supremum or integral norm and (ii) the span of any Schauder basis in a Banach space with basis. In particular, this includes the span of the standard unit vectors in $l_{p}(\mathbf{N})(1 \leqslant p<\infty)$.

EXAMPLE 2. We may apply Theorem 1 with $S$ of arbitrary cardinality. In particular, since the $l_{p}$ sum $(1 \leqslant p<\infty)$ of disjoint dense operator ranges produces such ranges, one can find injective operators $L_{i}$ mapping $l_{p}(S)$ to $l_{2}(S)(i=1,2)$ such that $R\left(L_{1}\right) \cap R\left(L_{2}\right)=0$ while each range is dense. Theorem 1 , with $p=2$, now shows that $R\left(L_{2}^{*} L_{1}\right)$ is unsupportable. One can argue similarly in $l_{p}(S)$ $(1<p<\infty)$.

EXAMPLE 3. If (1) is replaced by

$$
\operatorname{dim}\left[M \cap R\left(T^{*}\right)\right]=n \in \mathbf{N},
$$

the proof of the theorem shows that one can construct closed bounded convex sets whose support points form a nonempty set of dimension $n$. In particular, such a set fails to be the closed convex hull of its support points.

All the subspaces constructed via Theorem 1 will have uncountable codimension in $E$. This follows because $T(B)$ has too many support points. The next construction remedies this defect. Recall first that a space is barrelled if every closed bounded convex set with nonempty core has nonempty interior. 
THEOREM 2. Every reflexive separable Banach space E contains a dense unsupportable subspace which has countable codimension and is therefore barrelled.

Proof. Let $T: E^{*} \rightarrow c_{0}(\mathbf{N})$ be injective, continuous, and linear with proper dense range [13]. Let $P:=\operatorname{sp}\left(e_{n}\right)$ in $l_{1}(\mathbf{N})$ and let $M$ be a dense subspace of $l_{1}(\mathbf{N})$ with $M \cap P=0$, as can be guaranteed by a diagonal extraction process. Extend $T^{*}(M)$ to a supplement, $N$, for $T^{*}(P)$. Now consider $C:=T^{*}(B) \cap N$ in $N$, where $B:=\left\{x \in l_{1}(\mathbf{N})\|x\|_{1} \leqslant 1\right\}$. Since $B$ is $\omega^{*}$-compact, so is $T^{*}(B)$. Hence, $T^{*}(B)$ is closed in $E$ and $C$ is closed in $N$. Argue as in Theorem 1. If $0 \neq \Phi$ supports $C$ at $T^{*}(b)=c$ in $N$, then since $T^{*}(B \cap M) \subset C \cap N$ we have

$$
\begin{aligned}
0 & =\sup \langle\Phi, C \cap N-c\rangle \geqslant \sup \left\langle\Phi, T^{*}(B \cap M)-T^{*}(b)\right\rangle \\
& =\sup \langle T(\phi), B \cap M-b\rangle=\sup \langle T(\phi), B-b\rangle .
\end{aligned}
$$

Thus, $\|T \Phi\|=\langle T(\Phi), b\rangle=\|T(\Phi)\|\|b\|_{1}$ and $0 \neq b \in l_{1}(\mathbf{N})$ must support the ball in $c_{0}(\mathbf{N})$ of radius $\|T(\Phi)\|$ at $T(\Phi) \neq 0$. Hence, $b \in P$ and $c \in T^{*}(P) \cap N=0$. Thus, $b=0$, which is a contradiction. Clearly, $N$ is countable codimension and necessarily barrelled [18].

ExAmple 4. (a) In Theorem $2, T^{*}(B)$ provides an example of a convex set of a Banach space whose support points are countable dimensional. By the Bishop-Phelps theorem this is the smallest dimension one can achieve in an infinite-dimensional Banach space.

(b) Using the techniques of Theorem 2, dense unsupportable subspaces exist in any reflexive space which admits a proper dense injection into $c_{0}(S)$.

(c) A concrete version of Theorem 2 is provided by considering the inclusion of various spaces in $c_{0}(S)$. Then $T^{*}(B)$ is replaced by the $l_{1}(S)$ unit ball $B_{1}$, viewed, say, in $E:=l_{p}(S)(1<p<\infty)$ or in $E:=c_{0}(S)$. A direct argument shows that if $N$ is a subspace of $l_{1}(S)$ such that $N \cap V=0$, where $V$ is the span of the unit vectors, then $B_{1} \cap V$ is unsupportable in $V$ and $V$ is an unsupportable dense subspace of $E$.

A strong form of the Klee-Wilanski conjecture [17, 23] implies that every countable codimension subspace of a Banach space is Baire (second category in itself). But, recently, De Renya [20] has shown that there do, however, exist dense hyperplanes of first category in every separable Banach space of infinite dimension. Thus, the same holds in every W.C.G. space of infinite dimension. Nonetheless, we can show that the following theorem holds. The proof is a little tedious so we omit it.

THEOREM 3. Every reflexive separable Banach space contains a dense countable codimension unsupportable Baire subspace.

We complete this section by giving the standard example [14] which shows that any supportless normed space will possess a proper lower semicontinuous convex function with no subgradients. Such a function must, of course, be nowhere continuous. 
EXAMPLE 5. Let $C$ be a closed bounded convex subset of a normed space $E$ and let $0 \neq x_{0} \in E$. Define $f(x):=\min \left\{t \in \mathbf{R} \mid x+t x_{0} \in C\right\}$. Then $f$ is lower semicontinuous and convex, and since $f$ has no global minimum, any subgradient for $f$ at $x$ is nonzero. Letting $c:=x+f(x) x_{0}$ and noting that for any $y \in C, f\left(y-f(x) x_{0}\right) \leqslant$ $f(x)$, if $x^{*}$ is a subgradient of $f$ at $x$, then $x^{*}(y-c) \leqslant f\left(y-f(x) x_{0}\right)-f(x) \leqslant 0$. Thus, $x^{*}(y) \leqslant x^{*}(c)$ for all $y \in C$, so $x^{*}$ is a nonzero support for $C$ at $c$.

\section{Open questions.}

1. Is every incomplete normed space unsupportable?

From the previous results it would seem that, if not, one can ask the following:

2. Is there a supportable dense hyperplane (or finite codimension subspace) of a Banach space?

Closely related, but possibly distinct, is

3. Must the support points of a closed convex subset $C$ of a Banach space meet every hyperplane (or finite codimension affine subspace) which intersects the boundary of $C$ ?

Indeed, it is shown in [2] that the answer to 3 is positive for closed finite-codimension affine subspaces. Also, one can show that in a reflexive space the support points of a closed bounded convex set are weakly connected.

4. If $E$ is supportable does every closed bounded convex subset $C$ of $E$ have its support points dense in the boundary of $C$ ?

Dually, one can ask:

5. If every closed bounded convex subset of $E$ has a support functional, must the support functionals actually be dense in the dual?

By the converse to the dual Bishop-Phelps theorem, this is equivalent to 1 .

More limited questions include:

6. Does every Banach space possess a dense unsupportable subspace? Does $l_{\infty}(\mathbf{N})$ ?

7. Does every Banach space possess a closed bounded symmetric convex set with empty interior and with zero a nonsupport point?

It is a nontrivial fact that such a subset of $l_{\infty}(S)$ cannot be weak-star closed [4].

In light of our constructions one is led to ask:

8. Does every Banach space admit essentially disjoint dense operator ranges? Does $l_{\infty}(\mathbf{N})$ ?

Recall that a Banach space $E$ is W.C.G. (weakly compactly generated) if it admits a weakly compact densely generating set. Such spaces admit injections from $E$ and $E^{*}$ into $c_{0}(S)$. Moreover, $E$ is W.C.G. if and only if one can find a reflexive space $R$ and a dense linear injection $T$ of $R$ into $E$ [8]. Since such dense injections played a central role in Theorems 1 and 2, it makes sense to specialize the previous questions to a W.C.G. or reflexive setting.

Given our examples, we ask:

9. If $E$ is supportable, does every proper convex lower semicontinuous function have a nonempty (or densely defined) subgradient set?

In [23] Treiman has shown that, in a Banach space, the (Clarke) tangent cone of a closed set contains the topological limit inferior of the (Bouligand) contingent cones nearby. One can show that this result fails in any unsupportable space.

10. Does Treiman's theorem characterize Banach space? 


\section{REFERENCES}

1. E. Bishop and R. R. Phelps, A proof that every Banach space is subreflexive, Bull. Amer. Math. Soc. 67 (1961), 97-98.

2. The support functionals of a convex set, Convexity, Proc. Sympos. Pure Math., Vol. 7, Amer. Math. Soc., Providence, R. I., 1963, pp. 27-35.

3. J. M. Borwein and R. O'Brien, Tangent cones and convexity, Canad. Math. Bull. 19 (1976), 257-261.

4. Convex proximal sets with nonsurjective projections (preprint).

5. N. Bourbaki, Topological vector spaces, Hermann, Paris, 1958, p. 158.

6. A. Bronsted and R. T. Rockafellar, On the subdifferentiability of convex functions, Proc. Amer. Math. Soc. 16 (1966), 605-611.

7. F. H. Clarke, Optimization and nonsmooth analysis, Canad. Math. Soc. Monographs and Advanced Texts, Wiley, New York, 1968.

8. J. Diestal, Geometry of Banach spaces-selected topics, Springer-Verlag, Berlin and New York, 1975.

9. I. Ekeland, On the variational principle, J. Math. Anal. Appl. 47 (1974), 324-353.

10. __ Nonconvex minimization problems, Bull. Amer. Math. Soc. (N. S.) 1 (1979), 443-474.

11. I. Ekeland and G. Lebourg, Generic Frechet-differentiability and perturbed optimization problems in Banach spaces, Trans. Amer. Math. Soc. 244 (1976), 193-216.

12. P. A. Fillmore and J. F. Williams, On operator ranges, Adv. in Math. 7 (1971), 254-281.

13. S. Goldberg and A. H. Kruse, The existence of compact linear maps between Banach spaces, Proc. Amer. Math. Soc. 13 (1962), 808-811.

14. R. B. Holmes, Geometric functional analysis, Springer-Verlag, New York, 1975.

15. V. Klee, Extremal structure of convex sets. II, Math. Z. 69 (1958), 90-104.

16. On a question of Bishop and Phelps, Amer. J. Math. 85 (1963), 95-98.

17. V. Klee and A. Wilansky, Research problems, \#13, Bull. Amer. Math. Soc. 72 (1966), 656.

18. M. Levin and S. Saxon, Every countable codimensional subspace of a barrelled space is barrelled, Proc. Amer. Math. Soc. 29 (1971), 91-96.

19. N. Peck, Support points in locally convex spaces, Duke Math. J. 38 (1971), 271-278.

20. J. A. De Renya, Dense hyperplanes of first category, Math. Ann. 249 (1980), 111-114.

21. F. Sullivan, $A$ characterization of complete metric spaces, Proc. Amer. Math. Soc. 83 (1981), 345-346.

22. __ Ordering and completeness of metric spaces, Nieuw Arch. Wisk. 24 (1981), 178-193.

23. A. Todd and S. Saxon, A property of locally convex Baire spaces, Math. Ann. 206 (1973), 23-34.

24. J. S. Treiman, Characterizations of Clarke's tangent and normal cones in finite and infinite dimensions, Nonlinear Anal. 7 (1983), 771-783.

25. H. Tuy, A fixed point theorem including a hybrid inwardness-contraction condition, Math. Nachr. 102 (1981), 271-275.

Department of Mathematics, Dalhousie University, Halifax, Nova Scotia, Canada B3H 4H8 